\title{
Avaliação do Potencial Alelopático de Substâncias Isoladas em SEMENTES DE ARATICUM (Annona crassiflora) ${ }^{1}$
}

\author{
Allelophatic Potential of Substances Isolated from Annona crassiflora Seeds
}

\author{
INOUE, M.H. ${ }^{2}$, SANTANA, D.C. ${ }^{2}$, VILHENA, K.S.S. ${ }^{3}$, SOUZA FILHO, A.P.S..$^{4}$, GUILHON, G.M.S.P. ${ }^{3}$, \\ POSSAMAI, A.C.S. ${ }^{2}$, SILVA, L.E. ${ }^{5}$ e DALLACORT, R. ${ }^{2}$
}

\begin{abstract}
RESUMO - Este estudo visou identificar substâncias biologicamente ativas e o potencial alelopático de esteroides glicosilados, provenientes das sementes de Annona crassiflora. O isolamento dos esteroides glicosilados foi realizado com a separação dos constituintes químicos do extrato bruto de acetato de etila em coluna cromatográfica, sendo a completa elucidação estrutural por meio de espectroscopia de RMN ${ }^{1} \mathrm{H}$. Testes de germinação com as espécies Euphorbia heterophylla e Ipomoea grandifolia foram conduzidos em câmaras tipo BOD a $25^{\circ} \mathrm{C}$ e fotoperiodo de 12 horas, monitorados durante 10 dias com avaliação diária, nas concentrações de 0, 20, 40, 80 e $100 \mathrm{mg} \mathrm{L}^{-1}$ dos materiais isolados. Utilizando as mesmas concentrações, os experimentos de desenvolvimento de radícula e hipocótilo foram mantidos em câmara de germinação a $25{ }^{\circ} \mathrm{C}$ e fotoperíodo de 24 horas, com avaliação no décimo dia. Após o extrato de acetato de etila ser submetido ao fracionamento, verificou-se a presença de sinais característicos de fitoesteróis no espectro do $\mathrm{RMN}{ }^{1} \mathrm{H}$, resultando em uma mistura de estigmasterol glicosilado e sitosterol glicosilado. Os resultados indicam que as substâncias estigmasterol e sitosterol não proporcionaram inibição na germinação e no índice de velocidade de germinação (IVG) de E. heterophylla e I. grandifolia. Por outro lado, estigmasterol e sitosterol interferiram no desenvolvimento de radicula e hipocótilo de E. heterophylla.
\end{abstract}

Palavras-chave: estigmasterol, sitosterol, germinação, desenvolvimento.

\begin{abstract}
This work aimed to identify substances and the allelopathic potential of steroidal glycosides, isolated from the seeds of Annona crassiflora. Isolation of steroidal glycosides was carried out with the separation of chemical components of ethyl acetate extract in a chromatographic column and identified by 1 H NMR. Germination tests with Euphorbia heterophylla and Ipomoea grandifolia were carried out in $B O D$ Germinator at $25^{\circ} \mathrm{C}$ and $12 \mathrm{hr}$ photoperiod, monitored for 10 days at concentrations of $0,20,40,80$ and $100 \mathrm{mg} \mathrm{L}^{-1}$ of the materials isolated. Using the same concentrations, experimental radicle and hypocotyl development was maintained at $25^{\circ} \mathrm{C}$ with $24 \mathrm{hr}$ photoperiod for 10 days. After the ethyl acetate extract was submitted to fractioning, the presence of characteristic signs of steroids in the $1 H$ NMR spectrum was verified, providing a mixture of stigmasterol and sitosterol glycoside. The results indicate that the substances stigmasterol and sitosterol did not inhibit germination and germination velocity index (SIG) of E. heterophylla and I. grandifolia. However, both substances affected radicle and hypocotyl development of E. heterophylla.
\end{abstract}

Keywords: stigmasterol, sitosterol, germination, development.

Recebido para publicação em 20.11.2009 e na forma revisada em 12.11.2010.

2 Dep. de Agronomia, Universidade do Estado de Mato Grosso, Rodovia MT 358, Km 07, Caixa Postal 238, 78300-000 Tangará da Serra-MT, <miriamhinoue@ atmail.com>; ${ }^{3}$ Faculdade de Química, Universidade Federal do Pará, Belém-PA, Brasil; ${ }^{4}$ Laboratório de Agroindústria, Embrapa Amazônia Oriental, Belém-PA, Brasil; ${ }^{5}$ Laboratório de Química em Produtos Naturais, Universidade Federal do Mato Grosso, Cuiabá-MT. 


\section{INTRODUÇÃO}

O ecossistema de cerrado - considerada a mais rica biodiversidade de todas as savanas do mundo - necessita de mais estudos para se compreender a grande fonte de substâncias que esse bioma pode fornecer (Costa et al., 2008), uma vez que, entre os inúmeros compostos produzidos por sua flora, alguns podem ser úteis para o manejo de plantas daninhas.

Com o desafio de constituir um controle efetivo das plantas daninhas que se enquadrem nas exigências da preservação e qualidade dos recursos naturais (Souza Filho et al., 2005a), o isolamento de substâncias alelopáticas, bem como sua identificação, proporciona grande avanço no fornecimento de alternativas sustentáveis no controle de plantas daninhas em áreas agrícolas do cerrado.

Sabe-se que em condições de campo não há como separar os efeitos atribuídos à competição da alelopatia, pois a influência das plantas sobre outras espécies, em sua vizinhança, é uma combinação complexa de interferência da competição e das reações químicas alelopáticas (Olofsdotter et al., 1999; Kato-Noguchi et al., 2002). Vários estudos já foram desenvolvidos com o intuito de isolar e identificar substâncias alelopáticas de diferentes plantas (Inderjit, 1996; Duke et al., 2000; Kato-Noguchi, 2003), visando complementar os métodos tradicionais de manejo de plantas daninhas, por meio da redução do uso de herbicidas utilizando plantas que liberam substâncias tóxicas (alelopatia), reduzindo ou até mesmo inibindo totalmente seu desenvolvimento (Gomide, 1993). Nesse sentido, plantas da família Annonaceae podem ser uma ótima alternativa para produzir efeitos alelopáticos, por apresentarem atividades de herbicidas sobre diversas plantas daninhas (Leboeuf et al., 1982; Inoue et al., 2009).

Estudo fitoquímico realizado por Gonçalves et al. (2009) com a madeira de Annona crassiflora resultou na obtenção de dois alcaloides: aterospermidina e liriodenina. Este último possui atividades antimicrobiana e tripanosomicida; já o alcaloide aterospermidina possui atividade contra células de hepatoma humano (Gonçalves et al., 2009). Do mesmo modo, ao avaliar o extrato bruto preparado com sementes de A. crassiflora,
Santana et al. (2009) verificaram que determinadas substâncias naturais interferiram na germinação e no desenvolvimento de Brachiaria brizantha, Euphorbia heterophylla e Ipomoea grandifolia. Relatos disponíveis na literatura fazem com que esse gênero se destaque pelas diversas classes estruturais encontradas, como alcaloides, amidas, diterpenos, esteroides, flavonoides e acetogeninas (Pontes et al., 2004), úteis na produção de biodefensivos.

Diante da necessidade de estudos que visem isolar, identificar e, posteriormente, manipular moléculas com propriedades alelopáticas, este trabalho objetivou isolar e identificar substâncias biologicamente ativas das sementes de $A$. crassiflora, bem como avaliar o potencial alelopático dessas substâncias sobre a germinação e o desenvolvimento de E. heterophylla e I. grandifolia.

\section{MATERIAL E MÉTODOS}

Sementes de A. crassiflora foram coletadas no município de Nova Marilândia-MT, em área de cerrado, secas em estufa de circulação forçada de ar por 72 horas, à temperatura de $40^{\circ} \mathrm{C}$, e posteriormente trituradas em moinho tipo Wiley.

$\mathrm{O}$ isolamento e a identificação dos esteroides glicosilados do extrato bruto de acetato de etila foram realizados no Laboratório de Agroindústria da Embrapa Amazônia Oriental, localizado em Belém-PA. Os demais procedimentos foram conduzidos no Laboratório de Entomologia da Universidade do Estado de Mato Grosso, campus de Tangará da Serra-MT.

Inicialmente foram utilizados $3,0 \mathrm{~kg}$ de material moído (sementes), para obtenção dos esteroides glicosilados provenientes do extrato bruto de acetato de etila de $A$. crassiflora. O procedimento aplicado foi a sequência dos solventes com polaridade crescente, na seguinte ordem: éter de petróleo < etanol. O período de cada extração foi de seis horas ininterruptas, em aparelhos de Soxhlet. Após finalizar a extração, o extrato bruto de etanol de $A$. crassiflora foi fracionado com outros solventes, utilizando o método de Cromatografia de Coluna Filtrante (CCF). O método foi realizado com os solventes na ordem de polaridade crescente (diclorometano $<$ acetato de etila $<$ metanol), para 
obtenção dos respectivos extratos brutos de A. crassiflora.

$\mathrm{O}$ isolamento dos esteroides glicosilados foi realizado com a separação de componentes químicos do extrato acetato de etila por Cromatografia de Coluna por Via Úmida (CCVU), chamada de "R1" (Figura 1).

Para o preparo da CCVU, a coluna cromatográfica foi forrada com algodão, de forma que a sílica, ao ser adicionada, não se perdesse. A sílica foi adicionada juntamente com o solvente (hexano) como fase estacionária da coluna, na proporção de 1:15. Após a adição da sílica, foi preparada a pastilha do extrato, na proporção de 1:3. Portanto, utilizaram-se $10 \mathrm{~g}$ do extrato bruto de acetato de etila, $150 \mathrm{~g}$ de sílica para fase fixa da coluna e $30 \mathrm{~g}$ de sílica para compor a pastilha do extrato.

Em seguida, iniciou-se a separação, utilizando os solventes em ordem de polaridade crescente, a fim de retirar as substâncias contidas no extrato. Cada sistema utilizou $300 \mathrm{~mL}$ na combinação dos solventes, que foram coletados na seguinte ordem: 1) hexano (100\%), 2) hexano/acetato de etila (95:5), 3) hexano/ acetato de etila $(75: 25)$, 4) hexano/acetato de etila $(50: 50), 5)$ acetato de etila $(100 \%)$, 6) acetato de etila/metanol (90:10), 7) acetato de etila/metanol (80:20), 8) acetato de etila/ metanol (70:30), 9) acetato de etila/metanol (60:40), 10) acetato de etila/metanol (50:50) e 11) metanol (100\%), totalizando 32 amostras.

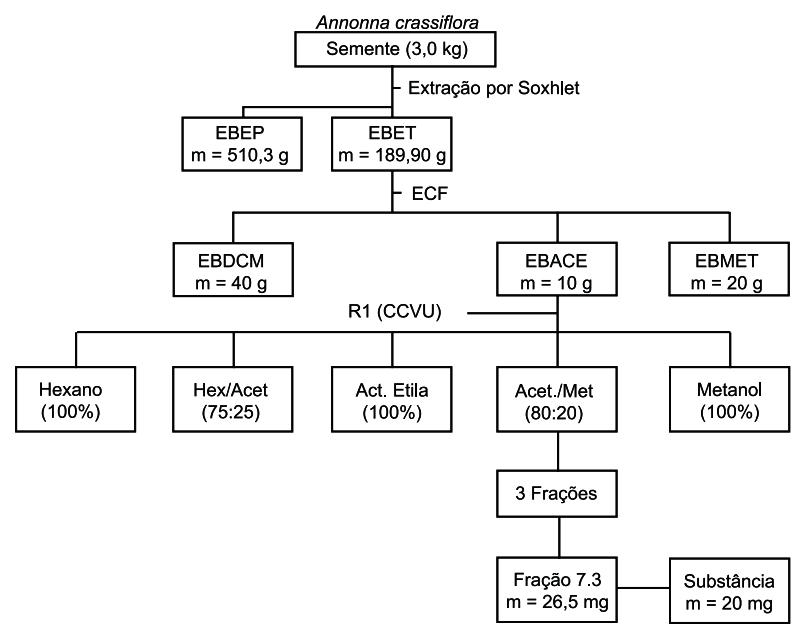

Figura 1 - Representação esquemática do processo de isolamento e purificação das substâncias presentes no extrato de acetato de etila, preparado com sementes de $A$. crassiflora.
Após ser concentrada em temperatura ambiente, cada amostra foi solubilizada com pequena quantidade de solvente, conforme a sua polaridade. Em seguida, com ajuda de capilares, esse material foi aplicado em placas de Cromatografia de Camada Delgada - CCD (ALUMINUM BACKED TLC, SÍLICA GEL, HARD LAYER, F-254, SAI). As placas de CCD foram ativadas em estufa com temperatura de $100{ }^{\circ} \mathrm{C}$ durante uma hora. As placas foram eluídas em sistemas de solventes em polaridade crescente, reveladas com sulfato cérico [Ce $\left.\left(\mathrm{SO}_{4}\right)_{2} \cdot \mathrm{H}_{2} \mathrm{O}\right]$ e queimadas em estufa a $100{ }^{\circ} \mathrm{C}$.

As placas reveladas foram comparadas, e aquelas consideradas semelhantes, reunidas em uma amostra somente para identificação. A fração 7.3 revelada na CCD com acetato de etila/metanol (80:20) (sólido branco de massa igual a $26,5 \mathrm{mg}$ ) foi analisada por meio de RMN ${ }^{1} \mathrm{H}$ e identificada como uma mistura dos esteroides glicosilados (estigmasterol glicosilado e sitosterol glicosilado). A confirmação da identificação estrutural das substâncias foi feita a partir da comparação dos dados obtidos com aqueles encontrados na literatura para essas substâncias (Santos et al., 2004; Silva et al., 2006).

Os bioensaios de germinação e desenvolvimento foram realizados com o estigmasterol glicosilado e sitosterol glicosilado, obtidos no extrato da fração $7: 3$. Os testes de germinação foram conduzidos em placas de Petri de $9,0 \mathrm{~cm}$ de diâmetro, previamente limpas com álcool $70 \%$, forradas com duas folhas de papel-filtro esterilizadas em autoclave em temperatura de $127^{\circ} \mathrm{C}$ e $1 \mathrm{~atm}$. Cada placa recebeu 3,0 mL de extrato da fração 7:3 diluída em metanol, nas concentrações de $0,20,40,80$ e $100 \mathrm{mg} \mathrm{L}^{-1}$. Após a evaporação do solvente presente no extrato em temperatura ambiente, 25 sementes das espécies E. heterophylla ou I. grandifolia foram distribuídas dentro de cada placa. Os experimentos foram conduzidos em câmara de germinação (BOD) a $25^{\circ} \mathrm{C}$ e fotoperíodo de 12 horas, monitorada durante 10 dias com avaliação diária. Dessa maneira, considerouse semente germinada aquela em que a radícula apresentou $2 \mathrm{~mm}$ de comprimento, eliminando-a em seguida. $O$ índice de velocidade de germinação (IVG) foi obtido conjuntamente com os testes de germinação, conforme estabelecido por Maguire (1962). 
Os experimentos de desenvolvimento de radícula e hipocótilo de E. heterophylla e I. grandifolia foram conduzidos avaliando-se as mesmas concentrações do teste de germinação, também em placas de Petri. Foram acondicionadas três sementes pré-germinadas com $2 \mathrm{~mm}$ de comprimento, as quais foram mantidas em BOD a $25{ }^{\circ} \mathrm{C}$ com fotoperiodo de 24 horas, com avaliação no décimo dia após a montagem do experimento, medindo-se o comprimento da radícula e do hipocótilo. Todos os tratamentos receberam em cada placa igual volume de água destilada, mantendo-se o volume inicial de $3,0 \mathrm{~mL}$, assim como o tratamento de $0 \mathrm{mg} \mathrm{L}^{-1}$ (testemunha).

O delineamento experimental foi o inteiramente casualizado, com quatro repetições. Os resultados foram submetidos à análise de variância, e as médias, comparadas pelo teste de Scott-Knott a 5\% de probabilidade (SAEG, 1997).

\section{RESULTADOS E DISCUSSÃO}

O extrato etanólico proveniente das sementes de $A$. crassiflora foi submetido à partição com solventes. O extrato acetato de etila, após submetido ao fracionamento por cromatografia em coluna via úmida (CCVU), forneceu uma mistura das substâncias estigmasterol glicosilado (ES5) e sitosterol glicosilado (ES6) (Figura 2). Similarmente a esse estudo, Chaves (1997) verificou a presença da mistura de estigmasterol e sitosterol em A. squamosa, comprovando a presença dessas substâncias também nesta espécie da família das Annonaceae.

No espectro do $\mathrm{RMN}{ }^{1} \mathrm{H}$ de ES5 e ES6 (Figura 2), pode-se verificar a presença de sinais característicos de hidrogênios de esteroide. Observa-se um singleto largo em $\delta_{\mathrm{H}}$ 5,34, relativo ao hidrogênio $(\mathrm{H}-6)$ ligado ao carbono olefinico C-6 de um esteroide; dois duplos dupletos, um em aproximadamente $\delta_{\mathrm{H}} 5,07$ e outro em aproximadamente $\delta_{\mathrm{H}} 5,14$, característicos de hidrogênios ligados a carbonos olefínicos (C-22 e C-23) da ligação dupla presente na cadeia lateral do estigmasterol; um multipleto em $\delta_{\mathrm{H}} 3,47-3,55$, correspondente a hidrogênio ligado a carbono oximetínico C-3; além dos demais sinais, referentes aos hidrogênios ligados aos carbonos dos grupos metílicos, metilênicos e metínicos.

A identificação do sitosterol pode ser justificada pela forte intensidade dos sinais nas
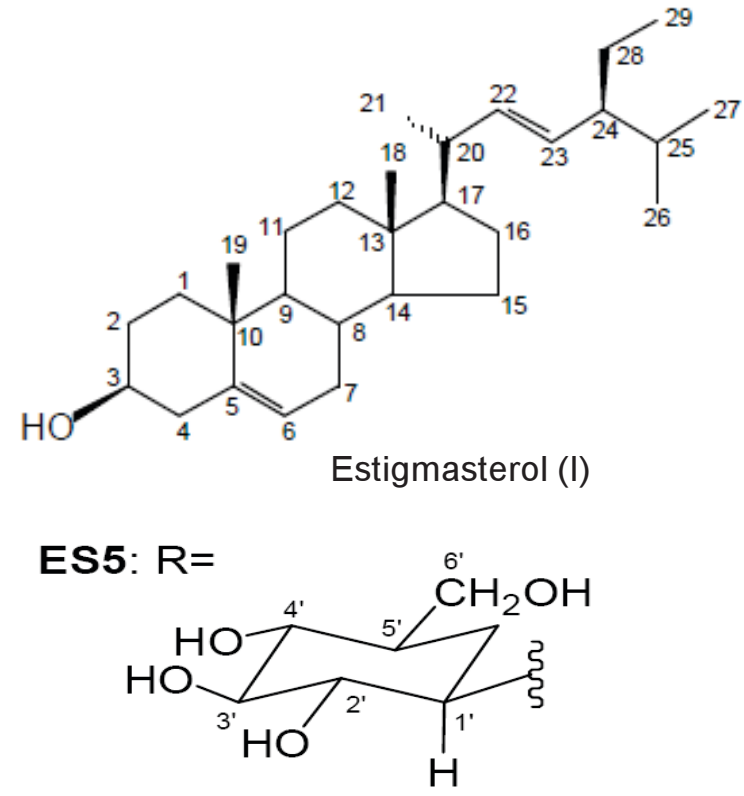
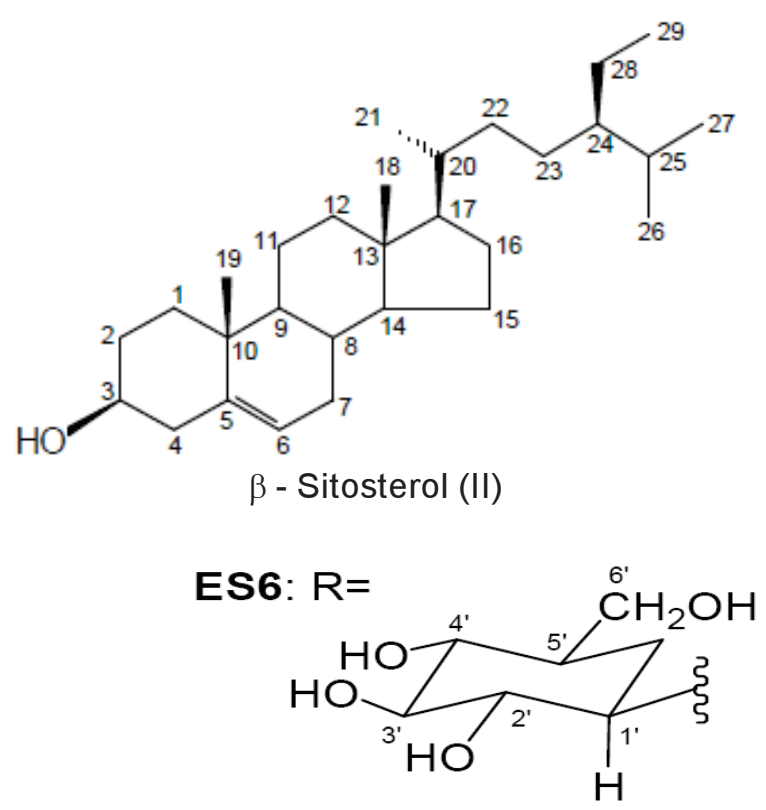

Figura 2 - Substâncias isoladas do extrato bruto de acetato de etila de A. crassiflora. São os esteroides glicosilados: estigmasterol glicosilado (ES5) e sitosterol glicosilado (ES6). 
regiões das metilas em $\delta_{\mathrm{H}} 0,66$ e entre $\delta_{\mathrm{H}} 0,92$ 0,84 (Figura 2). Além dos deslocamentos anteriormente citados, relativos às estruturas do estigmasterol e do sitosterol, são observados ainda sinais que caracterizam a presença de uma unidade de carboidrato. Observam-se um dupleto largo em $\delta_{\mathrm{H}} 4,58(\mathrm{~J}=12,3 \mathrm{~Hz})$ e um duplo dupleto $\mathrm{em}_{\mathrm{H}} 4,42(\mathrm{~J}=12,0$ e 6,0$)$, atribuídos a H-6'a e H-6'b, respectivamente. Foram verificados um multipleto sobreposto em $\delta_{\mathrm{H}} 4,3-4,2$ e outro em $\delta_{\mathrm{H}} 4,0-3,9$, relativos aos hidrogênios H-1', H-2', H-3', H-4' e H-5' (Figura 2). Os sinais que foram mencionados podem ser observados no espectro de $\mathrm{RMN}{ }^{1} \mathrm{H}$ obtido da fração 7.3 , conforme analisado em literatura (Hung \& Yen, 2001; Falcão, 2003; Rocha \& Silva et al., 2007).

Nos bioensaios com esteroides glicosilados (fração 7.3), verificou-se que essas substâncias não proporcionaram inibição na germinação $\mathrm{e}$ no IVG de E. heterophylla e I. grandifolia (Tabela 1). Esse fato indica que a fração de esteroides glicosilados não provocou nenhuma redução na germinação em relação à testemunha, evidenciando não ter ocorrido efeito alelopático sobre as espécies avaliadas no que se refere à germinação. Provavelmente, esses aleloquímicos apresentam diferentes efeitos alelopáticos em relação à fase em que se encontra a planta testada. Resultados semelhantes foram obtidos por Alves et al. (2003), quando testaram o efeito alelopático de alcaloides glicosilados de Solanum crinitum sobre a germinação de alface nas concentrações de 0,100, 200, 400 e $800 \mathrm{mg} \mathrm{L}^{-1}$, sendo observado que as substâncias não proporcionaram interferência na germinação de alface, independentemente da concentração utilizada.
Embora a variação no efeito alelopático possa estar associada aos fatores de concentração na substância analisada (Souza Filho et al., 2005a), não houve diferença significativa dentro do intervalo de concentrações avaliados (20 a $100 \mathrm{mg} \mathrm{L}^{-1}$ ) nos bioensaios de desenvolvimento de E. heterophylla e I. grandifolia (Tabela 2). Portanto, a concentração de aleloquímicos nas concentrações estudadas também não foi capaz de alterar o desenvolvimento de radícula e hipocótilo de I. grandifolia, em relação à testemunha (Tabela 2).

Por outro lado, todas as concentrações de esteroides glicosilados alteraram tanto o desenvolvimento de radícula como o hipocótilo de E. heterophylla (Tabela 2), evidenciando a sensibilidade no desenvolvimento dessa espécie às substâncias avaliadas. Esse resultado indica o efeito alelopático de estigmasterol glicosilado e sitosterol glicosilado sobre a espécie testada, ressaltando-se que o desenvolvimento de $E$. heterophylla foi a fase mais sensivel aos aleloquímicos testados, ao contrário da germinação (Tabela 1). Da mesma forma, em comparação à germinação das sementes, dados obtidos por Souza Filho et al. (2005b) indicaram que o crescimento da radícula foi o parâmetro mais sensivel aos efeitos da substância 4,5-diidrobulmenol A. Virtuoso (2008), ao avaliar a fração das cascas de Erythrina velutina, em que foram isolados compostos de estigmasterol e sitosterol, observou que essas substâncias foram capazes de inviabilizar o pleno desenvolvimento de Lactuca sativa, comprovando o efeito alelopático desses aleloquímicos.

Tabela 1 - Efeito de esteroides glicosilados provenientes das sementes de A. crassiflora sobre a germinação e IVG de E. heterophylla e I. grandifolia

\begin{tabular}{|c|c|c|c|c|}
\hline Concentração & \multicolumn{2}{|c|}{ Radícula $(\mathrm{cm})$} & \multicolumn{2}{c|}{ Hipocótilo (cm) } \\
\hline$\left(\mathrm{mg} \mathrm{L}^{-1}\right)$ & E. heterophylla & I. grandifolia & E. heterophylla & I. grandifolia \\
\hline 0 & $1,3 \mathrm{Aa}$ & $1,7 \mathrm{Aa}$ & $1,7 \mathrm{Aa}$ & $2,0 \mathrm{Aa}$ \\
\hline 20 & $0,2 \mathrm{Ba}$ & $0,7 \mathrm{Aa}$ & $0,1 \mathrm{Ba}$ & $1,2 \mathrm{Aa}$ \\
\hline 40 & $0,4 \mathrm{Ba}$ & $1,2 \mathrm{Aa}$ & $0,6 \mathrm{Ba}$ & $0,7 \mathrm{Aa}$ \\
\hline 80 & $0,3 \mathrm{Bb}$ & $1,2 \mathrm{Aa}$ & $0,1 \mathrm{Bb}$ & $1,6 \mathrm{Aa}$ \\
\hline $\mathrm{CV}(\%)$ & $0,4 \mathrm{Ba}$ & $0,8 \mathrm{Aa}$ & $0,2 \mathrm{Ba}$ & $1,2 \mathrm{Aa}$ \\
\hline
\end{tabular}

Médias seguidas da mesma letra, maiúscula na coluna e minúscula na linha, não diferem pelo teste de Scott-Knott a 5\% de probabilidade. 
Tabela 2 - Efeito de esteroides glicosilados provenientes das sementes de A. crassiflora sobre o desenvolvimento de radícula e hipocótilo de E. heterophylla e I. grandifolia

\begin{tabular}{|c|c|c|c|c|}
\hline Concentração & \multicolumn{2}{|c|}{ Radícula $(\mathrm{cm})$} & \multicolumn{2}{c|}{ Hipocótilo (cm) } \\
\hline$\left(\mathrm{mg} \mathrm{L}^{-1}\right)$ & E. heterophylla & I. grandifolia & E. heterophylla & I. grandifolia \\
\hline 0 & $1,3 \mathrm{Aa}$ & $1,7 \mathrm{Aa}$ & $1,7 \mathrm{Aa}$ & $2,0 \mathrm{Aa}$ \\
\hline 20 & $0,2 \mathrm{Ba}$ & $0,7 \mathrm{Aa}$ & $0,1 \mathrm{Ba}$ & $1,2 \mathrm{Aa}$ \\
\hline 40 & $0,4 \mathrm{Ba}$ & $1,2 \mathrm{Aa}$ & $0,6 \mathrm{Ba}$ & $0,7 \mathrm{Aa}$ \\
\hline 80 & $0,3 \mathrm{Bb}$ & $1,2 \mathrm{Aa}$ & $0,1 \mathrm{Bb}$ & $1,6 \mathrm{Aa}$ \\
\hline 100 & $0,4 \mathrm{Ba}$ & $0,8 \mathrm{Aa}$ & $0,2 \mathrm{Ba}$ & $1,2 \mathrm{Aa}$ \\
\hline $\mathrm{CV}(\%)$ & & & 28,2 \\
\hline
\end{tabular}

Médias seguidas da mesma letra, maiúscula na coluna e minúscula na linha, não diferem pelo teste de Scott-Knott a 5\% de probabilidade.

Os resultados indicam que as substâncias estigmasterol glicosilado e sitosterol glicosilado, isoladas no extrato bruto de acetato de etila de sementes de A. crassiflora, reduziram o desenvolvimento de radícula e hipocótilo da espécie E. heterophylla, evidenciando efeito alelopático sobre essa planta daninha.

\section{AGRADECIMENTOS}

Ao CNPq e à FAPEMAT, pelo suporte financeiro e auxílio com bolsas.

\section{LITERATURA CITADA}

ALVES, C. C. F. et al. Atividade alelopática de alcalóides glicosilados de Solanum crinitum Lam. Flor. Amb., v. 10, n. 1, p. 93-97, 2003

CHAVES, M. H. Análise de extratos de plantas por CCD: uma metodologia aplicada à disciplina "química orgânica". Química Nova, v. 20, n. 5, p. 560-562, 1997.

COSTA, J. V. M. et al. Raízes de plantas nativas do cerrado, gêneros Matayba e Serjania, da família do guaraná (Sapindaceae), apresentam princípios ativos contra o fungo causador da brusone em trigo. In: SIMPÓSIO NACIONAL DE CERRADO, 9.; SIMPÓSIO INTERNACIONAL DE SAVANAS TROPICAIS, 2., 2008, Brasília. Anais... Brasília: Embrapa Cerrado, [2008]. CD-ROM.

DUKE, S. O. et al. Natural products as sources of herbicides: current status and future trends. Weed Res., v. 10, n. 1, p. $99-111,2000$

FALCÃO, D. Q. Estudo químico e farmacológico de quatro espécies de Hyptis do Estado do Rio Grande do Sul. 2003. 148 f. Dissertação (Mestrado em Ciências Farmacêuticas - Universidade Federal do Rio de Janeiro, Rio de Janeiro, 2003
GOMIDE, M. B. Potencialidades alelopáticas dos restos culturais de dois cultivares de cana-de-açúcar (Saccharum sp.), no controle de algumas plantas daninhas. 1993. $96 \mathrm{f}$. Tese (Doutorado em Fitotecnia) Escola Superior de Agricultura Luiz de Queiroz, Piracicaba, 1993.

GONÇALVES, M. A.; LARA, T. A.; PIMENTA L. P. S. Alcalóides oxaporfínicos da madeira de Annona crassiflora Mart. In: REUNIÃO ANUAL DA SOCIEDADE BRASILEIRA DE QUÍMICA, 29., 2009, Águas de Lindóia Anais... São Paulo: Sociedade Brasileira de Química, [2009]. CD-ROM

HUNG, C. Y:; YEN, G. C. Extraction and identification of antioxidative components of Hsiantsao (Mesona procumbens Hemsl.). Lebensm Wiss Techn., v. 34, p. 306-11, 2001 .

INOUE, M. H. et al. Extratos aquosos de Xylopia aromatica e Annona crassiflora sobre capim-marandu (Brachiaria brizantha) e soja. Sci. Agr., v. 10, n. 3, p. 245-250, 2009.

INDERJIT. Plant phenolics in allelopathy. Bot. Rev., v. 62, p. 186-202, 1996.

KATO-NOGUCHI, $\mathrm{H}$. et al. Isolation and identification of a potent allelopathic substance in rice root exudates. Physiol. Plant., v. 115, p. 401-405, 2002

KATO-NOGUCHI, H. Isolation and identification of an allelopathic substance in Pisum sativum. Phytochemistry, v. 62, p. 1141-1144, 2003.

LEBOEUF, $M$. et al. The phytochemistry of the Annonaceae Phytochemistry, v. 21, p. 2783-2813, 1982.

MAGUIRE, J. D. Speed of germination-aid in selectionevaluation for seedling emergence and vigour. Crop Sci., v. 2, n. 1, p. 176-199, 1962 
OLOFSDOTTER, M. et al. Weed-suppressing rice cultivars does allelopathy play a role? Weed Res., v. 39, p. 441-454, 1999.

PONTES, A. F.; BARBOSA, M. R. V.; MAAS, P. J. M. Flora paraibana: Annonaceas Juss. Acta Bot. Bras., v. 18, n. 2, p. 281-293, 2004.

ROCHA E SILVA, H. et al. Constituintes químicos das cascas do caule de Cenostigma macrophyllum: ocorrência de colesterol. Química Nova, v. 30, n. 8, p. 1877-1881, 2007.

SAEG - Sistema para Análises Estatísticas: versão 7.0. Viçosa,MG: Fundação Arthur Bernardes, 1997.

SANTANA, D. C. et al. Reação do extrato bruto de acetato de etila da Annona crassiflora sobre espécies de plantas daninhas. In: SIMPÓSIO IBEROAMERICANO DE PLANTAS MEDICINAIS, 4., 2009, Cuiabá. Anais... Itajaí: Rede Iberoamericana de Estudo e Aproveitamento Sustentável da Biodiversidade Regional de Interesse Farmacêutico, [2009] CD-ROM.
SANTOS, A. R. et al. Constituintes polares das folhas de Machaonia brasiliensis (Rubiaceae). Química Nova, v. 27, n. 4, p. 525-527, 2004.

SILVA, V. C. et al. Constituintes fenólicos e terpenóides isolados das raízes de Andira fraxinifolia (Fabaceae). Química Nova, v. 29, n. 6, p. 1184-1186, 2006.

SOUZA FILHO, A. P. S.; FONSECA, M. L; ARRUDA, M. S. P. Substâncias químicas com atividades alelopáticas presentes nas folhas de Parkia pendula (Leguminosae) Planta Daninha, v. 23, n. 4, p. 565-573, 2005a.

SOUZA FILHO, A. P. S.; LOBO, L. T.; ARRUDA, M. S. P Atividade alelopática em folhas de Tachigali myrmecophyla (Leg. - Pap.). Planta Daninha, v. 23, n. 4, p. 557-564, $2005 b$.

VIRTUOSO, S. Estudo fitoquímico e biológico das cascas de Erythrina velutina Willd. - Fabaceae (Leguminosae Papilionoideae). 2008. 111 f. Dissertação (Pós-Graduação em Ciências Farmacêuticas ) - Universidade Federal do Paraná, Curitiba, 2008 